\title{
THE INCLUSION OF A FINITE CAPTURE TIME IN THE NUMERICAL SIMULATION OF QUANTUM EFFECT DEVICES.
}

\author{
M. Gault, H. Matsuura*, K. Furuya*, P.Mawby and M.S. Towers \\ Department of Electronic Engineering \\ University College of Swansea \\ Singleton Park Swansea \\ SA2 8PP \\ U.K.
*Department of Electronic Engineering
Tokyo Institute of Technology
O-Okayama Meguro-Ku
Tokyo 2-12-1
Japan

\begin{abstract}
A simple numerical method is presented to include the phenomenon of a finite quantum well capture time in the numerical simulation of quantum effect devices. If the time taken to leave the quantum well (through tunnelling) is sufficiently short the electron states in the quantum well will remain relatively unoccupied due to the finite scattering time from the three dimensional continuum into the two dimensional states. A numerical formulation is presented which models this phenomenon by using an effective Fermi function for the occupancy of the two dimensional states, enabling the method to be used in conjunction with general purpose device simulators. The method is applied to a simple tunnel barrier to show the generality of the model.
\end{abstract}

\section{INTRODUCTION}

In the operation of single or double heterobarriers the accumulation of electrons at the heterointerface can have significant effects on the performance of the device. The principle of operation is shown in figure 1. As the bias is increased across the heterobarrier a potential induced accumulation layer forms at the heterointerface. Electrons in the accumulation layer have their energies quantised in the direction of propagation.

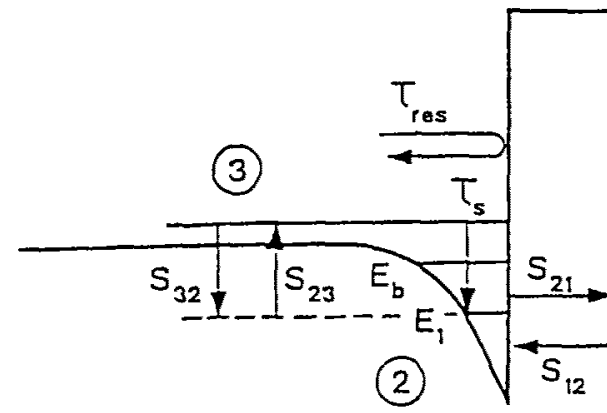

Figure 1: Principle of operation

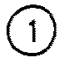


To model this phenomenon requires accurate knowledge of the electron concentration in the accumulation layer. As the bias is increased above zero volts it is no longer valid to assume that the electrons in the accumulation layer are in thermal equilibrium. If the time required to enter the $2 \mathrm{D}$ states is much longer than the time required to leave the $2 \mathrm{D}$ states then the $2 \mathrm{D}$ states will remain relatively unoccupied. A numerical model for such structures is presented next.

\section{NUMERICAL MODEL.}

In order to simulate the steady state characteristics of the device the following equations must be solved self consistently. To obtain the electrostatic potential throughout the device Poisson's equation must be solved (assuming majority carrier operation)

$$
\nabla \cdot(\varepsilon \nabla \varphi)=-q\left(-n+N_{D}\right)
$$

where $\varepsilon$ is the composition dependent permittivity, $q$ is the electronic charge, $\varphi$ is the electrostatic potential, $\mathbf{n}$ is the electron concentration, and $N_{D}$ is the concentration of ionised donors. The electron density is calculated from the addition of $2 \mathrm{D}$ electrons in bound energy states and $3 \mathrm{D}$ thermal electrons.

$$
n=\underbrace{\left.\sum_{i=1}^{n} \frac{m^{*} k_{B} T}{\hbar^{2}} f_{i}^{*} \ln \left[1+\exp \left(\frac{E_{f}-E_{i}}{k_{B} T}\right)\right] \varphi_{i}\right|^{2}}_{2 D}+\underbrace{N_{c} F_{1 / 2}\left(\frac{E_{f}-E_{b}}{k_{B} T}\right)}_{3 D}
$$

where $f$ is the non-equilibrium modification factor, $k_{B}$ is Boltzmann's constant, $E_{f}$ is the quasi-Fermi energy, $N_{c}$ is the effective density of states, $E_{b}$ represents the energy above which electrons are unbound (usually the top of the quantum well), $F_{1 / 2}$ represents the Fermi integral, $\mathrm{m}^{*}$ is the electron effective mass and $\hbar$ is Planck's constant $h / 2 \pi$. $E_{i}$ and $\varphi_{i}$ are the quantised energy levels and wavefunctions respectively, calculated from the effective mass Schrödinger equation,

$$
\left(\frac{-\hbar^{2}}{2} \nabla\left(\frac{1}{m^{*}} \nabla\right)+V\right) \varphi_{i}=E_{i} \varphi_{i}
$$

where $\mathrm{V}$ is the potential defining the quantum well (the conduction band edge).

To calculate the tunnelling current through the barrier a modified Tsu-Esaki formula [1] is used. In the case of an accumulation layer there are 2 components to the current density, a two dimensional component $J_{2 D}$ and a three dimensional component $J_{3 D}$. 


$$
\begin{aligned}
& J_{2 D}=\sum_{i=1} \frac{4 \pi q m^{*} k_{B} T}{h^{3}} \int^{E_{0}} T(E) \cdot \ln \left(\frac{\left(1+\exp \left(\frac{E_{f}-E}{k_{B} T}\right)\right)^{f_{i}^{*}}}{\left(1+\exp \left(\frac{E_{f}-E-q V_{b i}}{k_{B} T}\right)\right)}\right) d E \\
& J_{3 D}=\frac{4 \pi q m^{*} k_{B} T}{h^{3}} \int_{E_{0}}^{\infty} T(E) \cdot \ln \left(\frac{\left(1+\exp \left(\frac{E_{f}-E}{k_{B} T}\right)\right)}{\left(1+\exp \left(\frac{E_{f}-E-q V_{b i}}{k_{B} T}\right)\right)}\right) d E
\end{aligned}
$$

where $T(E)$ is the energy dependent tunnelling probability through the barrier (obtained from a solution to (3)) and $V_{b i}$ is the voltage drop across the heterobarrier. Note that due to coupling between the quantum well and the collector the integral for the $2 \mathrm{D}$ current cannot be replaced by a summation.

The non-equilibrium factor $f$ is used to cope with the non-equilibrium of electrons and is derived as [2].

$$
\mathrm{f}^{*}=\frac{\frac{\tau_{\mathrm{mi}}}{\tau_{\mathrm{z}}} \mathrm{f}_{3}^{\circ} \mathrm{g}_{3}+\mathrm{T}_{21} \mathrm{f}_{1} \mathrm{~g}_{1}}{\frac{\tau_{\mathrm{ma}}}{\tau_{\mathrm{k}}} \mathrm{f}_{3}^{\circ} \mathrm{g}_{3}+\mathrm{T}_{21} \mathrm{f}_{2}^{0} \mathrm{~g}_{1}}
$$

$T_{21}$ represents the peak tunnelling probability through the barrier, $\tau_{\text {res }}$ represents the resident time of an electron above the quantum well, $\tau_{s}$ represents the scattering time into the well, f represents the occupancy probability and $g$ represents the density of states. The subscripts refer to figure 1 . The only unknowns are $\mathrm{f}_{1}$ and $\mathrm{g}_{1}$ and these can easily be calculated.

To model the effects of scattering on energy spectrum a simple method proposed by [3] is used. Lorentzian broadening of the tunnelling probability spectrum is assumed, the magnitude of which is calculated from the phase relaxation time, the time between phase altering events. In this way the tunnelling probability $\mathrm{T}(\mathrm{E})$ for any particular energy $E$ is modified according to

$T^{*}(E)=T(E)\left(\frac{\Gamma_{c}}{\Gamma}\right) \frac{1}{1+\left[\left(E-E_{r}\right) /(\Gamma / 2)\right]^{2}}$

where $T^{*}(E)$ is the modified transmission probability of the barrier at energy $E, E_{T}$ is the resonance energy of the well, $\Gamma$ is the total energy width due to coupling outside the quantum well and phase breaking inside the well, $\Gamma=\Gamma_{c}+\Gamma_{p}$ where $\Gamma_{c}$ and $\Gamma_{p}$ are the coupling width and the width caused by phase breaking respectively. The relationship between the width caused by phase breaking and the phase breaking time $\tau_{\mathrm{p}}$ is given by the uncertainty principle.

\section{APPLICATIONS}

Figure 2 a shows how the occupancy of the well decreases with decreasing width of barrier. In this example the scattering time into the well is taken to be lps in agreement with recent experimental results[4]. The 
result clearly indicates the importance of considering a finite scattering time in the case of narrow barriers. At barrier of widths of $8 \mathrm{~nm}$ and above the quantum well may be taken as fully occupied (within the bounds of the underlying Fermi-Dirac statistics) but below $8 \mathrm{~nm}$ this is clearly not so. At barrier widths of $2 \mathrm{~nm}$ and below the occupancy of the two dimensional states in the quantum well drops rapidly after a small applied voltage and plays only a small part in the current operation of the device.

Figure $2 b$ shows how the occupancy of the well varies for various scattering times into the quantum well. The barrier width in this example is taken to be $4 \mathrm{~nm}$. At a scattering time of $1 \mathrm{ps}$ the occupancy of the well drops linearly with applied voltage. As the scattering time into the quantum well increases so the occupancy of the well decreases, as would be expected.
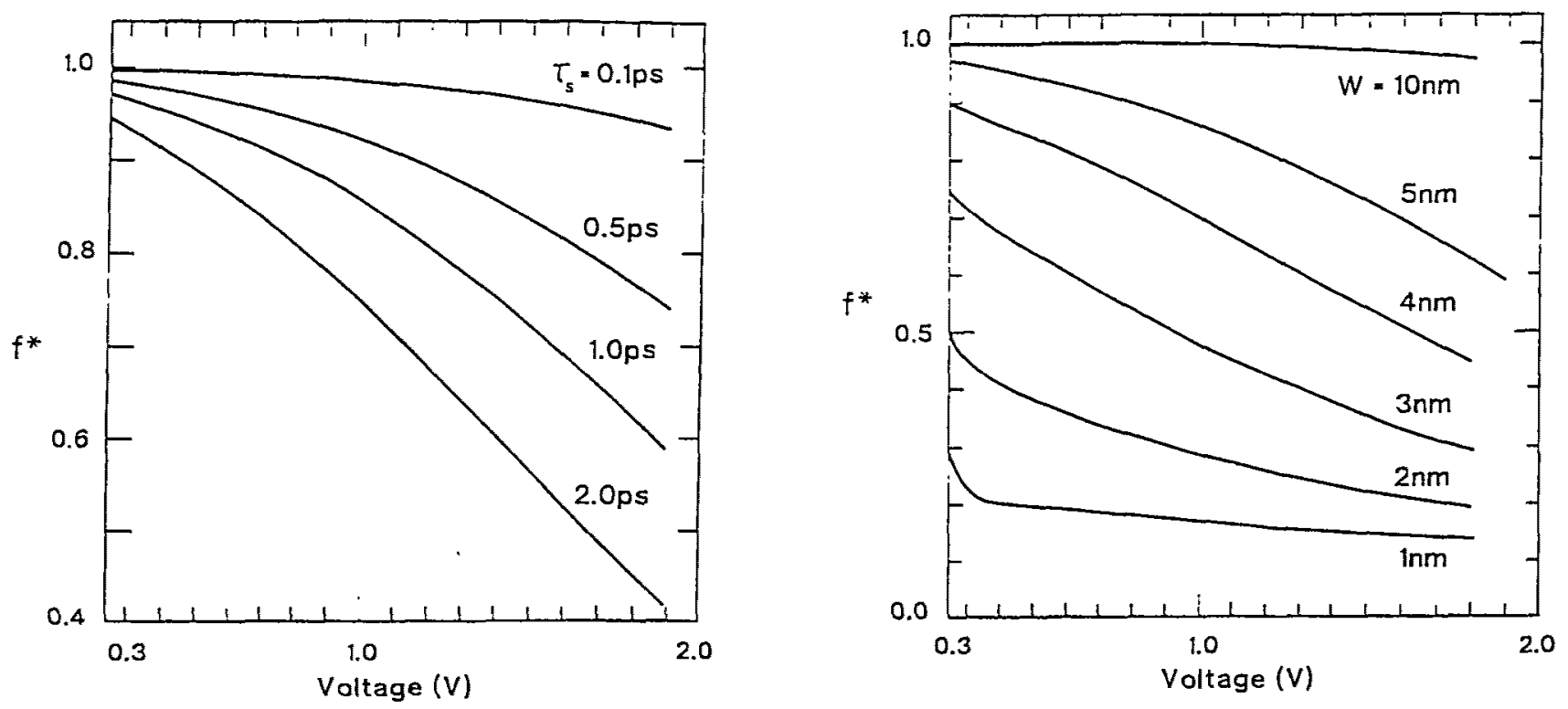

Figure 2 Occupation probability vs. a) scattering time and b) barrier thickness

\section{CONCLUSIONS}

In summary a simple formalism has been derived to calculate the reduced occupancy of a quantum well due to a finite capture time which is both easy to calculate and to implement within existing device simulators. The results show the importance of including the finite capture time in tunnel barrier simulation for narrow barriers where the occupancy can be reduced to around $10 \%$ of its equilibrium value at a moderately high applied voltage.

\section{REFERENCES}

1. $\quad$ R. Tsu and L. Esaki, Appl. Phys. Lett., 22, 562 (1973).

2. M. Gault, H. Matsuura, K. Furuya, P.Mawby and M.S. Towers, To be published in solid-state electronics (1994).

3. M. Jonson and A. Grincwajg, Superlattices and Microstructures, 12, 453 (1992).

4. M.R.X. Barros, P.C. Becker, D. Morris, B. Deveaud, A. Regreny, F. Beisser, Physical Review $B, 47,10951$ (1993). 